\title{
Chemical compound formation and diffusion - as parallel processes in grain boundary
}

\begin{abstract}
The new model of grain boundary diffusion is developed taking into account the simultaneous grain boundary diffusion (in Fisher-Gibbs approximation) and chemical interaction, which leads to the formation of the molecules $M N$ type in binary system. The evaluation is made of "reaction" effect on the rate of the whole process.
\end{abstract}

Keywords: chemical compound, kinetic, diffusion, fisher's model, grain boundaries
Volume 4 Issue 2 - 2020

\author{
Petelin AL, Bokstein BS, Novikov AA, \\ Novikova EA \\ Department of Physical Chemistry, National University of \\ Science and Technology “MISIS”, Russia
}

\begin{abstract}
Correspondence: Petelin AL, Department of Physical Chemistry, National University of Science and Technology “MISIS", Leninsky Pr. 4, Moscow, RU-I I 9049, Russia, Tel

+79057 I 2 1023, Email alexander-peteli@yandex.ru
\end{abstract}

Received: January 29, 2020 | Published: March II, 2020

\section{Introduction}

The Fischer's model ${ }^{1}$ is the base of the standard description of the diffusion along grain boundary (GB) with leakage to grain bulk. Various versions of calculation diffusant concentration field were realized: for self diffusion and hetero diffusion, with a grain boundary segregation ${ }^{2,3}$ and formation of atomic complexes in $\mathrm{GB},{ }^{4-6}$ for various temperature $\operatorname{areas}^{7}$ and various structural types of GBs. ${ }^{8}$ It may seem strange, but the model describing influence of chemical processes in $\mathrm{GB}$, which bind a part of diffusing atoms, on the rate of their moving in GB wasn't developed. Though the experimental fact of grain boundary diffusion (GBD) delay due to precipitation of nanoparticles in GB is well-known, it is usually connected with the idea that fine particles which are formed in GB reduce the section of boundary and diffusive flux. The direction of this investigation is the development the Fisher's model of GBD taking into account simultaneous chemical interaction of diffusive and matrix atoms in GB. The events of this kind - parallel carrying out two processes with participation of the same atoms - are well-known in heterogeneous kinetics. ${ }^{9}$ As a typical example of those processes it can be considered the steel nitration process.

\section{Model}

Let's consider a bicrystal sample consists of substance $M$ - two grains with grain boundary in the middle. The thickness of GB is $\delta$ (Figure 1). The substance $N$ is diffusing impurity which can diffuse along GB. We propose there are three processes during GBD at the same time: two correspond to Fisher's model - advanced diffusion of $N$ atoms in GB and leakage to the bulk - and the third, connected with forming in GB of a chemical compound of $M_{p} N_{q}$ type. Further we will restrict our consideration to the simplest case of $M N$ compound. For description of the first two processes we use Fisher's model: GB is located normally to external surface, the substance $N$ moves from a surface where its concentration $c_{s}=$ const, along GB (on $y$ axis), with diffusion coefficient $D_{b}$, and also run from GB into grain bulk (along $x$ axis) with coefficient $D<<D_{b}$ (Figure 1). Let's keep all assumptions of Fisher's model, corresponding to the B - regime according to Harrison /7/: width of GB is $\delta<<\sqrt{D t}$, where $t$ is the time of diffusion annealing, we will neglect direct diffusion from the surface to the bulk. Let's describe a process of formation of $M N$ molecule in GB as a chemical reaction:

$$
M+N \stackrel{k^{\prime}}{\longrightarrow} M N
$$

$N$

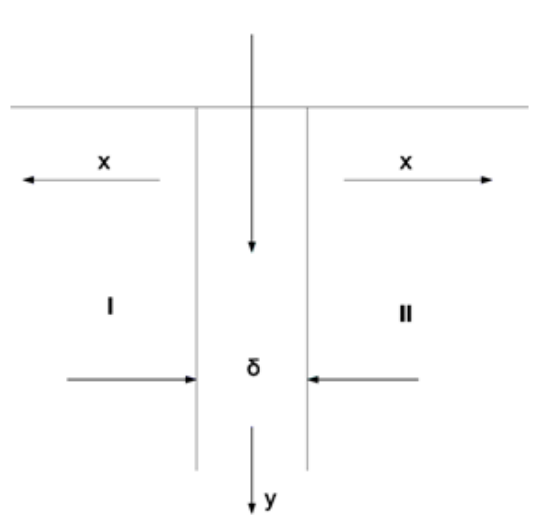

Figure I N diffusion in GB with leakage to the bulk, $\delta$ - the GB thickness.

According to the theory of simple collisions /9/ the reaction rate $v$ can be described as:

$$
v=-\frac{d c_{b}}{d t}=\dot{k}^{\prime} c_{b}(M) c_{b}(N)
$$

where $c_{b}(M)$ and $c_{b}(N)$ - are the concentrations of $M$ and $N$ in GB, and $k^{\prime}$ - constant of the process rate (1). In a bulk we neglect formation of $M N$ compound. As $c_{b}(M)=$ const, instead of (2) it is possible to write:

$$
v=k c_{b}(N)
$$


where $k=k^{\prime} c_{b}(M)$. Taking into account reaction (3), Fisher's equations will look like:

$$
\begin{gathered}
\frac{\partial c(x, y, t)}{\partial t}=D \frac{\partial^{2} c(x, y, t)}{\partial x^{2}} \\
\frac{\partial c_{b}(y, t)}{\partial t}=D_{b} \frac{\partial^{2} c_{b}(y, t)}{\partial y^{2}}+\left.\frac{2}{s \delta} D \frac{\partial c(x, y, t)}{\partial x}\right|_{x= \pm \frac{\delta}{2}-k c_{b}}
\end{gathered}
$$

Here $c$ is the concentration of the element $N$ in a grain bulk. The third term of sum in the right part of equation (5) describes reduction of concentration of diffusing element in GB due to the binding of part of $N$ atoms - this is a new element of the model. The second term of the sum, as well as at Fisher's model, describes leakage of $N$ atoms from GB to the bulk. $s$ is a segregation factor

$$
s=\left.\left(\frac{c_{b}}{c}\right)\right|_{x= \pm \delta / 2}
$$

\section{Results and discussion}

Solving the equation (4), and, according to the Fisher's quasistationary condition, assuming $\partial c_{b} / \partial t=0$, we receive instead of the equation (5)

$$
\frac{d^{2} c b}{d y^{2}}-\left(\frac{k}{D_{b}}+\frac{2 \sqrt{D}}{s \delta D_{b} \sqrt{\pi t}}\right) c_{b}=0
$$

The equation (7) differs from the usual Fisher's by the term $\left(\frac{k}{D_{b}}\right.$ ) which describes contribution of chemical interaction in GBD. The solution of the equation (7) looks like

$$
c_{b}=c_{s} \exp \left(-\frac{y}{L}\right)
$$

where

$$
\begin{gathered}
L=\frac{L_{k} L_{D}}{\left(L_{k}^{2}+L_{D}^{2}\right)^{1 / 2}} \\
L_{k}^{2}=\frac{D_{d}}{k}
\end{gathered}
$$

and

$$
L_{D}^{2}=\frac{s \delta D_{b} \sqrt{\pi t}}{2 \sqrt{D}}
$$

In these equations $-L_{D}$ is diffusion length or usual Fisher's length, i.e. distance on which concentration of diffusing substance decreases in " $e$ " times owing to GBD and outflow, and $L_{k}$ - kinetic length concentration decreases due to formation of a chemical compound $M N$. Linear dependence of $\ln c_{b}$ upon $y$ remains, but as $L_{k}^{2}+L_{D}^{2}>L_{D}^{2}$, diffusion at GB is slowed down. In accordance with the system (8-11) we can introduce two limiting regimes of GBD. In the first the rate of diffusion is controlled by atom movement in GB and leakage to the bulk (diffusion regime). In the second - by reaction of $M N$ molecule formation (kinetic regime). The border between these two regimes can be found from the condition $L_{k}=L_{D}$. We can transform $L_{D}$ from (11) to

$$
L_{D}^{2}=\sqrt{\pi} \alpha D_{b} t
$$

In (12) $\alpha=\frac{s \delta}{2 \sqrt{D t}}$ is a dimensionless parameter introduced by Le Claire ${ }^{11}$ For GBD in B-regime (according to Harrison /7/) $\alpha \leq 0,1$ We evaluate $L_{D}$ for $T=0,5 T_{m}, t=3,6 \cdot 10^{5} \mathrm{~s}$ and $\alpha \leq 0,1$. To estimate $D_{b}$ we use empirical rule ${ }^{12}$

$$
D_{b}=\exp \left(-\frac{E_{b}}{R T}\right)
$$

where $D_{b}=10^{-5} \mathrm{~m}^{2} \mathrm{~s}^{-1}$ and $E_{b}=9 R T_{m} \cdot{ }^{10}$ Consequently, $D_{b} \approx 1,5 \cdot 10^{-13} \mathrm{~m}^{2} \mathrm{~s}^{-1}, L_{D}^{2} \geq 10^{-10} \mathrm{~m}^{2}$ and $L_{D} \geq 10^{-5} \mathrm{~m}$. To evaluate $L_{k}$, we need the value of $k$, which is unknown. We use the next consideration. To form a molecule of compound $M N$, the solute atom $N$ has to jump and form the bond with solvent atom $M$. Other words it is necessary to overcome the potential barrier which is also unknown. Naturally, we assume that the rate constant $k$ is less, than the atom jump frequency $\left(\Gamma_{b}\right)$ in grain boundary. To evaluate the $\Gamma_{b}$ value we remember that in cubic crystals $D=\frac{a^{2}}{12} \Gamma$, where $a$ is inter atomic distance. For simple metals $a \approx 0,4 \mathrm{~nm}$ and $\Gamma_{b}=\frac{12 D_{b}}{a^{2}}=10^{7} \mathrm{~s}^{-1}$ (for $T=0,5 T_{m}$ ). (14). Equalizing $L_{k}$ from (10) and $L_{D}$ from (12) with $\alpha \leq 0,1$ and $a \approx$ $0,4 \mathrm{~nm}$ we recue

$$
k^{*}=\frac{2,4 \cdot 10^{20}}{\Gamma \cdot t^{2}}
$$

Where $k^{*}$ is the $k$-value which corresponds to $L_{k}=L_{D}$-the border between diffusion and kinetics regimes. One can see that increase of temperature and annealing time leads to decrease of $k^{*}$. Now we can examine $c_{b}$-dependence on the penetration depth using the system of equations $(8-11)$ with above evaluated values of $L_{k}, L_{D}$ and $k^{*}$, the results are shown on Figure 2. From Eq. (15) it follows that $k^{*}$ depends inversely on $\Gamma$ and $t^{2}$. For $t=100$ hours $=3,6 \cdot 10^{5} s k^{*}=2 \cdot 10^{10} / \Gamma$ and with $\Gamma=10^{7} \mathrm{~s}^{-1}$

$$
k^{*}=2 \cdot 10^{3} s^{-1}
$$

Naturally $k^{*}<\Gamma$.

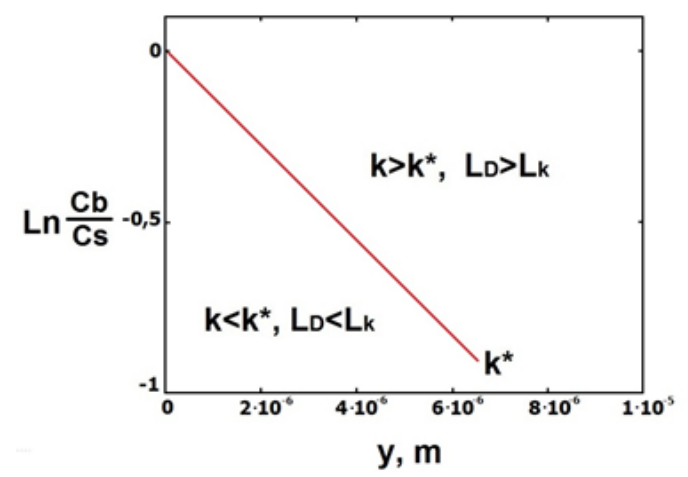

Figure $2 c_{b}$ - dependence on the penetration depth, the line corresponds to $k=k^{*}$ and $L_{k}=L_{D}$. The region above the line corresponds to diffusion, and below - to kinetic regimes. 


\section{Conclusion}

The simultaneous realization of GBD with cleavage to the bulk and "reaction" of molecule $M N$ type formation in binary system is discussed. It is shown that we can introduce two limiting regimes diffusion and kinetic. The estimations show that the formation of $M N$ molecule slows down GBD. Do not taking into account the kinetic correction, we obtain the data for $\mathrm{s}_{\mathrm{b}}$, which are less then true ones.

\section{Acknowledgments}

None.

\section{Conflicts of interest}

Author declares that there is no conflict of interest.

\section{References}

1. Fisher JC. Calculation of Diffusion Penetration Curves for Surface and Grain Boundary Diffusion. J Appl Phys. 1951;22(1):74-77.

2. Gibbs JB. Grain Boundary Impurity Diffusion. Phys Stat Sol. 1966;16:K27-K29.

3. Mishin Y, Herzig Chr. Grain boundary diffusion: recent progress and future research. Mat Sci and Enginiring. 1999;260:55.
4. Guttmann M. Grain boundary segregation, two dimensional compound formation, and precipitation. Met Trans. 1977;8A:1383-1401.

5. Bokstein BS, Esin VA, Rodin AO. Phys MM. 2010;109:316.

6. Esin V, Bokstein B, Rodin A. Concentration Profiles for Grain Boundary Diffusion in B-Regime with Regard to the Formation of Atomic Complexes in Grain Boundary. Def and Dif. Forum. 2011;309-310:29.

7. Harrison LG. Influence of dislocations on diffusion kinetics in solids with particular reference to the alkali halides. Trans Faraday Soc. 1961;57:1191.

8. Bokstein B. Structure and properties of internal interfaces in metals. M Nauka. 1988:272.

9. Panchenkov GM, Lebedev VP. Chemical kinetics and catalysis M Chemistry. 1074:592.

10. Bokstein BS, Mendelev MI. Short course of physical chemistry. MISiS. 2002:232.

11. Le Claire AD. The analysis of grain boundary diffusion measurements. Br J Appl Phys. 1963;14:351.

12. Kaur I, Mishin Y, Gust W. Fundamentals of Grain and Interphase Boundary Diffusion. Chichester, UK. Wiley; 1995. 